\title{
Dispositif de formation de sujets lecteurs enseignants du secondaire
}

\author{
Training device for secondary school teacher readers
}

doi: 10.18162/fp.2020.501

\section{R \\ ésumé}

Nous rendons compte, dans le présent article, de notre recherche doctorale, notamment du dispositif didactique que nous avons expérimenté avec de futurs enseignants de français (FEF) en formation dans une école supérieure du Gabon.

La séquence didactique, organisée en sept séances de cours, a été menée par un enseignant formateur. Nous avons observé et interprété l'investissement subjectif de huit participants volontaires, afin de comprendre comment mieux les former à la lecture littéraire et à son enseignement.

\section{Mots-clés}

Didactique du français, séquence didactique, formation des enseignants, sujet lecteur enseignant, lecture littéraire, rechercheformation, cercle de lecture, carnet de lecteur.

Abstract

In this article, we report on our doctoral research, in particular, the didactical device we experimented with future French teachers (FEF) in training a superior school of Gabon. The didactic sequence organized in seven course sessions was conducted by a teacher trainer. We observed and interpreted the subjective investment of eight volunteer participants to understand how to better train them in reading and teaching.

Keywords

French didactics, didactic sequence, training of teachers, subject teachers drive, literary reading, research training, reading circle, reading logbook.

\section{Introduction}

D'après les travaux sur la formation initiale des enseignants au Gabon (Makaya, 2013; Nguimbi, 2015) et les rapports des conseillers et inspecteurs pédagogiques (MEN et IPN, 2012), il en ressort que l'enseignement de la lecture littéraire est organisé autour d'activités d'analyse textuelle et que la subjectivité du lecteur est source de fausseté. Par ailleurs, d'après les recherches en didactique du français (Langlade et Fourtanier,2007; Rouxel et Langlade,2004), toute lecture est une expérience individuelle. Partant de ces analyses, nous avons axé notre recherche sur la formation des sujets lecteurs enseignants au Gabon pour mettre les apprenants au cœur de l'apprentissage (MEN, 2018). Après une présentation succincte de notre problématique et des concepts clés de notre recherche, le présent article rend compte de notre méthodologie à travers le dispositif expérimenté avec de futurs enseignants en formation à l'ENS. Puis, l'analyse des principaux résultats nous a conduite à dégager les limites de ce dispositif.

\section{Problématique}

\section{Remise en question des approches formalistes de la lecture}

Les approches formalistes de l'enseignement de la lecture ont fait l'objet de vives critiques du fait qu'elles occultent la participation effective des lecteurs (Langlade, 2004a). Dans le cas de l'enseignement du français au Gabon, il s'agit précisément de la lecture expliquée, héritée de l'approche historique, et de la lecture méthodique, héritée des approches formalistes. Bien que relevant d'approches théoriques différentes, au Gabon, ces deux méthodes reposent essentiellement sur un enseignement magistral des formes et des procédés littéraires. 
La lecture méthodique (préconisée par les programmes gabonais depuis 1995) passe par une explication formelle des processus narratifs ou stylistiques, le texte littéraire étant présenté comme un objet langagier clos et autosuffisant. La persistance de l'enseignement de la lecture méthodique est un obstacle à la prise en compte des lecteurs réels (Ahr, 2014; Deronne, 2011).

En effet, ces vingt dernières années, plusieurs recherches ont été menées sur l'enseignement de la lecture littéraire de la maternelle à l'université (Dufays, Gemenne et Ledur, 2005; Rouxel, 1996; Tauveron, 1999). Même si le concept de lecture littéraire fait encore débat, un certain consensus pratique a émergé (Louichon, 2011) concernant l'importance de prendre en compte l'activité du lecteur dans l'élaboration des significations du texte. Pour Langlade et Fourtanier (2007), il convient de montrer aujourd'hui tout l'intérêt didactique d'un renouvèlement des pratiques scolaires de la lecture. Puisque chaque lecteur complète l'œuvre selon sa subjectivité, il est nécessaire de comprendre comment les élèves lecteurs s'impliquent dans la reconfiguration du texte littéraire. Les lectures réelles des élèves et donc la dimension singulière, subjective de toute lecture sont devenues des objets d'intérêts centraux pour certains didacticiens de la littérature. La notion de « sujet lecteur » rend compte de ces préoccupations (Rouxel et Langlade, 2004).

\section{La lecture littéraire}

La lecture littéraire a fait l'objet de plusieurs définitions en didactique du français. Aujourd'hui, il est admis que le lecteur joue un rôle actif dans la construction du sens textuel et que tout texte peut ainsi être considéré comme le produit d'une lecture (Bayard, 1998; Ricœur, 1985). Depuis 2004, après la parution des actes du colloque sur le sujet lecteur, plusieurs didacticiens de la littérature étudient l'actualisation et la reconfiguration de l'œuvre lue. Pour Sauvaire, la lecture littéraire permet « la rencontre entre un texte pluriel et mobile et un lecteur pluriel et changeant, dont les multiplicités s'altèrent mutuellement » (2013, p. 64). Pour Émery-Bruneau, la lecture littéraire est envisagée comme «l'activité dynamique d'un lecteur inscrit dans une dialectique entre une lecture participative, soit une lecture subjective vécue de façon plutôt affective, et une lecture distanciée, soit une lecture des effets vécue de façon plus intellectualisée » (2011, p. 79). Il s'agit donc d'un processus dynamique de recherche du sens, où le lecteur oscille entre les valeurs liées aux référents (l'unité de sens, la conformité esthétique, la vérité) et les valeurs liées aux formes du message (la polysémie, la subversion, la fictionnalité). Elle rejoint la définition de Dufays et al. (2005), présentant la lecture littéraire comme un va-et-vient dialectique entre les postures de participation et de distanciation.

\section{Finalités de l'enseignement de la lecture littéraire}

D'après les programmes officiels sur l'enseignement du français au Gabon dont les objectifs sont restés les mêmes depuis 1995, il faut «former des hommes équilibrés, capables de raisonner, de se servir de leur cerveau qui n'est pas un sac qu'il suffit de bourrer, mais une valeur multiforme à découvrir, à soutenir et à développer " (MEN et IPN, 1995, p. 3). Autrement dit, l'enseignement du français ne se réduit pas à une transmission de savoirs, mais devrait être le lieu de la formation à la réflexion et à l'élaboration d'un jugement personnel. Aussi, si l'on veut que les enseignants de français ne réduisent pas leur activité à faire acquérir aux élèves un répertoire de références, il est indispensable de préciser les finalités auxquelles répond l'enseignement de la lecture littéraire. 
Aujourd'hui, la lecture littéraire, définie comme « un processus dialectique qui requiert implication (lecture subjective) et distanciation (lecture objective) de la part des sujets lecteurs » (Ahr, 2014, p. 13), invite à ménager un espace où la voix du sujet lecteur pourra se faire entendre et se nourrira de celles des pairs dans un va-et-vient dialectique. Cette conception dialectique de la lecture littéraire présente des enjeux didactiques avérés. D'après Dufays (2013), la notion de lecture littéraire a été élaborée pour inspirer l'action des enseignants et des élèves afin qu'ils produisent des lectures singulières. Selon nous, l'approche théorique de la lecture littéraire peut servir de cadre à la programmation des activités d'enseignement de la littérature et à la formation des sujets lecteurs.

Il reste que si l'école est le lieu de la formation de la personnalité (Dufays et al., 2005; MEN, 2018; Rouxel, 1996), les approches exploitées dans la classe de français au Gabon réduisent l'apprenant à un simple exécutant. Comme le montre le dernier rapport sur l'enseignement du français au Gabon (Assoumou-Mombe, 2011) et les travaux sur la formation initiale au Gabon (Makaya, 2013), les objectifs de construction de l'individu, visés par les Instructions officielles, ne sont pas atteints. C'est pourquoi nous nous sommes demandé comment former des sujets lecteurs enseignants.

\section{Enseigner la lecture littéraire}

Enseigner la lecture littéraire, c'est prendre en compte la subjectivité des lecteurs, susciter les interactions entre pairs face au texte et former des sujets lecteurs. À partir de l'idée du va-et-vient entre les postures de participation et de distanciation selon Dufays et al. (2005), et de l'activité du sujet lecteur selon Sauvaire (2013), nous allons former des sujets lecteurs enseignants, c'est-à-dire :

des enseignants de français qui ne se limiteront pas à être que des « animateurs de lecture passionnés » ou que des " guides interprétatifs », mais qui seront aussi des professionnels réfléchis, sensibles, outillés, capables de faire vivre aux élèves des démarches inductives qui les pousseront à trouver la source de leur sensibilité et de leur réflexivité en lecture littéraire pour mieux se comprendre et se développer comme sujets-lecteurs. (Émery-Bruneau, 2011, p. 80)

À partir du dispositif expérimenté durant cette recherche, nous formulons que la formation des sujets lecteurs enseignants est un préalable à l'enseignement du français.

\section{Les sujets lecteurs enseignants, une nécessité didactique}

Dans le cadre de notre recherche doctorale, nous postulons qu'aider les futurs enseignants de français à assumer leur rôle et leur statut de sujet lecteur peut contribuer à les former à l'enseignement de la lecture littéraire. Pour Ahr, un sujet lecteur est :

un sujet autonome, critique, conscient de ses gouts, capable d'exprimer un ressenti, une émotion, un jugement en se fondant sur sa lecture du texte, son expérience, ses lectures antérieures [...], capable également de confronter son expérience de lecture non seulement à celle de ses pairs, mais également à celle des multiples lecteurs l'ont précédé. (2014, p. 12)

Autrement dit, c'est un lecteur qui lit un texte avec toutes les ressources subjectives et culturelles dont il dispose. Nous considérons la formation des sujets lecteurs enseignants comme étant intrinsèquement liée à l'enseignement de la lecture littéraire. 
Comme le souligne Deronne (2011), il semble opportun de former des sujets lecteurs enseignants afin qu'ils comprennent comment se développe la relation de soi au texte et de soi aux autres afin de déterminer de quelle manière la lecture littéraire contribue à la formation de soi comme lecteur. Ces futurs enseignants, considérés comme des sujets lecteurs, pourraient par la suite prendre en compte la participation des élèves dans leurs pratiques d'enseignement.

\section{Des enseignants formés à la réflexivité}

L'éclairage des travaux de Rouxel et Langlade (2004), de Fourtanier, Langlade et Mazauric (2006), d'Émery-Bruneau (2010) et de Sauvaire (2013) confirme que la lecture subjective est une lecture réflexive. D'après Sauvaire $(2013,2015)$, la réflexivité, considérée comme une mise à distance et un retour sur soi, est liée à l'émergence de la subjectivité. Ainsi, enseigner la lecture littéraire subjective, c'est contribuer au développement de la réflexivité des lecteurs.

Former les enseignants à la réflexivité, c'est leur apprendre à interpréter un texte littéraire et les amener à se comprendre comme des sujets lecteurs. Cette mise à distance de sa propre lecture est favorisée par l'écriture et l'oral réflexif (Chabanne et Bucheton, 2002; Sauvaire, 2015). C'est pourquoi, afin de développer les capacités réflexives des FEF, nous avons alterné dans notre séquence didactique des activités de production orale et écrite autour du recueil de poèmes Les temps déchirés d'Hallnaut Engouang (2011). Il semblerait que certaines activités occasionnent plus que d'autres des pratiques langagières réflexives ${ }^{1}$. L'enseignant doit donc se penser comme sujet lecteur dans la classe afin dêtre conscient du rôle de la subjectivité dans la construction du sens. De cette manière, la formation des sujets lecteurs enseignants contribue à leur formation à l'enseignement de la lecture littéraire.

\section{Difficultés de la formation des sujets lecteurs enseignants}

La formation des sujets lecteurs enseignants reste un défi à relever dans une tradition scolaire orientée vers la construction d'une posture de lecture experte. Selon Langlade (2004b), la lecture experte focalise l'interprétation sur les caractéristiques esthétiques et les outils d'analyse. C'est pourquoi nous pensons que faire place au sujet lecteur enseignant dans les programmes de formation, c'est accepter en classe la lecture subjective des futurs enseignants, c'est-à-dire, faire place à leur imaginaire et au va-et-vient entre l'investissement personnel dans la lecture et la mise à distance du texte. Pour Massol et Shawky-Milcent (2011) qui mettent en évidence la persistance d'exercices traditionnels soutenant une écriture normée de la lecture, prendre en compte les lectures subjectives des lecteurs est perçu par certains comme une forme de non-respect des droits du texte. Malgré ces résistances, nous pensons que la formation des sujets lecteurs enseignants est une nécessité didactique pour mieux comprendre et enseigner la lecture littéraire.

\section{Méthodologie}

Notre méthodologie s'inscrit dans l'approche qualitative. Nous souhaitions porter une attention particulière à la subjectivité des lecteurs. C'est pourquoi nous avons choisi l'approche qualitative, car elle nous a permis de recueillir des données verbales permettant une démarche interprétative. Partant de notre objectif général qui est de comprendre les effets d'un dispositif d'enseignement d'une œuvre 
intégrale sur la formation des FEF du secondaire au Gabon, nous avons retenu la recherche-formation comme type de recherche. Il s'agit d'un type de recherche réalisée avec le chercheur, le formateur et les enseignants en formation initiale ou continue. Pendant la collecte des données, le processus de la recherche-formation nous a permis d'interagir avec le formateur et les futurs enseignants durant la réalisation du dispositif didactique.

\section{Présentation du dispositif}

Nous nous sommes inspirée de plusieurs dispositifs didactiques (Burdet et Guillemin, 2013; Fourtanier et al., 2006; Ouellet, 2012; Terwagne, Vanhulle et Lafontaine, 2003) pour réaliser cette séquence didactique. Ces dispositifs ont l'avantage d'intégrer des activités de lecture, d'oral et d'écriture dans le but de développer les compétences langagières et interprétatives des élèves et des enseignants en formation. Nous avons suivi une progression intégrant le principe du va-et-vient entre les lectures individuelles des textes et leurs interprétations par les lecteurs dans les cercles de lecture (CL) et les carnets de lecture. Le but de cette séquence est d'amener chacun à formuler une interprétation subjective et de développer la réflexivité des sujets lecteurs enseignants (SLE).

\section{Population cible}

Concernant notre échantillon, nous avons procédé à un échantillonnage non probabiliste par choix raisonné orienté par les critères de sélection suivants :

- être un FEF inscrit à l'ENS au Gabon;

- être en première année de formation à l'enseignement du français au secondaire.

Notre population cible était composée d'étudiants outillés sur les méthodologies des textes et la didactique des épreuves aux examens du secondaire. Nous avions un enseignant formateur ${ }^{2}$ (formé à la didactique du français) et 17 étudiants (Master, CAPES, CAPC) âgés de 18 ans minimum et de classe sociale moyenne, répartis de la façon suivante :

\section{Tableau 1}

Répartition des participants au projet

\begin{tabular}{l|l|l|l} 
Étudiants participant au projet & Niveau & Nombre de femmes & Nombre d'hommes \\
\hline Master & $1^{\text {re }}$ année & 6 & 8 \\
\hline CAPES & $2^{\mathrm{e}}$ année interne & 1 & 0 \\
\hline CAPC & $1^{\text {re }}$ année & 0 & 2
\end{tabular}

Nos données proviennent de l'analyse de la séquence didactique réalisée par l'enseignant formateur avec les huit FEF volontaires et de l'entretien semi-dirigé collectif que nous avons mené avec ces derniers.

La particularité de ces participants était qu'ils n'avaient jamais enseigné le français au secondaire, de plus, ils avaient été outillés sur la pratique de la lecture méthodique pour l'enseignement des textes littéraires. Partant de notre objectif général qui est de comprendre les effets d'un dispositif d'enseignement d'une 
œuvre intégrale sur la formation des FEF du secondaire au Gabon, notre démarche s'inscrit sans aucun doute dans les versants herméneutiques ${ }^{3}$ et praxéologiques ${ }^{4}$ des recherches en didactique de la littérature (Dufays, 2010). Nous avons donc interprété les données recueillies et nous les avons analysées en fonction de nos objectifs de recherche.

\section{Déroulement de la séquence didactique}

Nous avons réalisé la séquence didactique à partir du recueil de poèmes Les temps déchirés d'Hallnaut Engouang, écrivain gabonais. Cette séquence s'inscrit dans l'approche par compétences prescrite par le ministère de l'Éducation nationale qui vise à offrir une éducation centrée sur le développement des habiletés de l'apprenant, habiletés transférables d'une discipline à l'autre, et sur son implication dans son apprentissage. Nous avons organisé cette séquence autour d'activités orales et écrites encourageant les FEF à adopter des postures créative et réflexive (Bucheton, Alexandre et Jurado, 2014). Nous pensons que ces différentes activités participeront à la formation des SLE.

La séquence réalisée comportait sept séances de cours et une séance préliminaire. En accord avec les étudiants lors du recrutement des participants et de l'enseignant formateur, il a été retenu de tenir deux séances par jour en matinée, allant de $9 \mathrm{~h}$ à $10 \mathrm{~h}$, et de $11 \mathrm{~h}$ à $12 \mathrm{~h} 30$. La séquence s'est déroulée dans une salle de cours à l'ENS en juin 2016. Finalement, cette séquence initialement prévue en huit séances s'est déroulée en sept séances (car les séances 7 et 8 mentionnées dans notre projet de recherche ${ }^{5}$ ont pu être combinées), variant d'une heure à $1 \mathrm{~h} 30$ aux heures précédemment mentionnées.

Durant la séance préliminaire, l'enseignant formateur a effectué des mises au point théoriques sur la lecture littéraire. À partir d'un fascicule de textes qui leur a été remis à l'avance, les FEF ont livré leur compréhension des notions clés de la lecture littéraire. Cette séance s'est achevée par la distribution du recueil de poèmes Les temps déchirés d'Hallnaut Engouang et des carnets des lecteurs. Les sept séances suivantes ont donné lieu à la composition des cercles de lecture et à l'étude proprement dite du recueil de poèmes. Durant cette séquence didactique, le rôle de l'enseignant formateur a consisté à susciter l'implication des étudiants et à les accompagner dans la prise de conscience de soi comme lecteur. La séquence s'est achevée par une analyse individuelle dans les carnets d'un poème choisi par l'ensemble des participants ${ }^{6}$.

\section{Justification du dispositif}

Selon Ouellet (2012), un dispositif est un ensemble de moyens et d'activités mis en place par un enseignant dans le but de former les élèves. En considérant la lecture littéraire comme un processus interactionnel entre les lecteurs et les œuvres (Fourtanier et al., 2006), nous avons conçu un dispositif d'enseignement pour former des sujets lecteurs enseignants. Nous avons cibléles activités qui favorisaient la confrontation entre pairs et l'investissement subjectif des lecteurs. Ce dispositif doit permettre de :

- stimuler l'activité fictionnalisante des lecteurs révélatrice et productrice d'imaginaire (Langlade et Fourtanier, 2007);

- susciter des interactions entre pairs afin d'enrichir et de développer la singularité de chaque lecteur, car on s'enrichit et on se développe mutuellement (Ouellet, 2012; Sauvaire, 2013); 
- favoriser une attitude réflexive (Dufays, 2013; Ouellet, 2012; Sauvaire, 2013; Vanhulle, 2009) par un va-et-vient dans les activités de lecture et d'interprétation du texte initial;

- recueillir les textes singuliers des lecteurs (Tauveron, 2007; Vibert, 2013).

Nous avons réalisé ce dispositif à partir d'un recueil de poèmes que nous avons choisi en fonction de la charge émotive et de la liberté interprétative offerte par la poésie. La recherche en didactique du français souligne la nécessité de varier les activités des apprenants dans l'enseignement de la lecture littéraire (Ahr, 2014; Chabanne et Bucheton, 2002; Fourtanier et al., 2006). En réalisant un dispositif d'enseignement susceptible de stimuler l'activité subjective des lecteurs, nous voulons proposer une solution de rechange aux approches traditionnelles. Selon nous, la confrontation des lectures individuelles des enseignants en formation pourrait être une source d'enrichissement pour la construction des interprétations de chaque lecteur. C'est pourquoi, dans cette séquence didactique, nous avons combiné différentes activités (cercles de lecture, carnet de lecture, lecture individuelle et collective) permettant d'amener les FEF à acquérir des compétences interprétatives pour la production d'un texte singulier.

\section{Les instruments de collecte de données}

\section{Le carnet de lecture}

Selon Giasson, le carnet de lecture est « un outil de réaction aux textes » (2014, p. 133). C'est la raison pour laquelle dès la séance préliminaire, il a été remis à chaque participant un carnet de lecture qui a été utilisé durant les 7 séances de cours. Les étudiants l'ont utilisé de différentes manières. Certains y ont retranscrit l'intégralité des activités menées au fil des séances. Pour d'autres, le carnet a servi uniquement aux interprétations individuelles du recueil. Le carnet de lecture est apparu comme un moyen de garder une trace des lectures individuelles des lecteurs et surtout un moyen de prendre en compte ce que font les lecteurs.

Dans cette recherche, nous considérons le carnet de lecture comme un instrument de collecte de données. Présenté comme un espace permettant de rendre compte de son activité de lecture (Ahr, 2013; Giasson, 2014), le carnet de lecture est une voie d'accès au texte singulier du lecteur. Les données collectées au moyen de cet instrument permettent de prendre connaissance des effets de cette séquence sur les interprétations des FEF. Il est non seulement un support à la mémoire, mais aussi une aide pour l'interprétation du texte. À ce jour, plusieurs travaux confirment son importance dans la formation des sujets lecteurs (Rouxel, 2013; Vibert, 2013). Ces auteures soutiennent que le carnet de lecture amène ses utilisateurs à adopter une attitude réflexive favorisant un va-et-vient entre leurs écrits et leurs lectures. Le carnet de lecture est aussi un outil réflexif. D’après Giasson (2014), le carnet de lecture permet de développer la réflexivité des lecteurs, car ceux-ci s'y réfèrent pour expliquer, analyser et évaluer une pratique. D'ailleurs, les textes des lecteurs qui ont été retranscrits et analysés dans cette recherche proviennent des carnets de lecture. Pour un des FEF, «c'est un moyen ou un outil qui nous permet de conserver les différentes réactions des lecteurs d'un texte littéraire »(Carnet, p. 1). 
En somme, il n'y avait pas de consignes d'utilisation des carnets. La liberté était donnée à chaque FEF d'en faire une utilisation personnelle. Nous avons remarqué que certains sont datés et organisés chronologiquement en fonction des séances, d'autres apparaissent comme de simples notes éparses. En dépit de cela, cet instrument nous a permis de prendre connaissance des effets du dispositif sur les interprétations des poèmes par les FEF (leurs capacités lectorales), et d'analyser les effets du dispositif sur la conception de la lecture littéraire construite par les FEF (l'appropriation d'une notion didactique).

\section{Les cercles de lecture}

Dès la séance 1, l'enseignant formateur a demandé aux FEF de constituer des cercles de lecture (désormais CL). Trois CL ont été constitués par les FEF en fonction de la disposition de salle de cours qui comptait trois rangées de tables-bancs. Un responsable a été désigné dans chaque cercle. Ce dernier avait pour rôle de faire la synthèse des activités menées dans le cercle. Ensuite, les FEF ont trouvé que l'on pouvait alterner les rôles dans le cercle. Ces différents cercles étaient représentatifs des différents niveaux des participants (CAPES, CAPC, Master).

Dans notre recherche, les CL constituent l'activité charnière de la séquence didactique. En effet, les discussions dans les CL ont pour objectif de favoriser l'investissement subjectif des lecteurs. Ces cercles ont été effectifs durant les séances 1,2, 4 et 5. D'après Terwagne et al., un CL est un « dispositif didactique au sein duquel les participants, rassemblés en petits groupes hétérogènes, apprennent à interpréter et à construire des connaissances à partir de textes littéraires ou d'idées » (2003, p. 7). Autrement dit, les cercles de lecture favoriseraient les interactions entre lecteurs et permettraient de mieux comprendre les textes.

Dans le cadre de cette séquence, les CL mis en place avaient pour objectifs de :

- susciter l'activité des sujets lecteurs;

- prendre en compte l'expérience subjective des lecteurs et leur maitrise du texte;

- permettre le dialogue entre pairs et apprendre à discuter pour construire ensemble de nouvelles idées sur le texte.

Pour l'ensemble des FEF de cette étude, les CL sont un dispositif à expérimenter en classe, car en suscitant les interactions entre pairs, ils donnent à chacun l'occasion de "dépasser ses peurs solitaires » (ENT, p. 13) et de "s'enrichir du point de vue des autres" (Carnet, p. 2). C'est donc un outil didactique qui nous semble adapté à la formation des sujets lecteurs enseignants.

\section{Présentation et analyse des données}

Dans cette partie, nous présentons l'analyse du discours des huit FEF étudiants en formation pour l'enseignement du français au secondaire au Gabon. Ces participants répondant à nos critères de sélection nous ont fourni des données variées pour mieux comprendre comment former les sujets lecteurs enseignants à partir du dispositif didactique réalisé. Les résultats des différents sujets proviennent des carnets de lecteur, des cercles de lecture et de l'entretien semi-dirigé intégralement retranscrits dans des verbatims. Tous les sujets se sont approprié le dispositif de façon singulière de 
telle sorte que leurs données apparaissent complémentaires (tableau 2) et nous permettent de voir les principales dimensions de la formation des SLE.

\section{Tableau 2}

Analyse des résultats par sujet

\begin{tabular}{|c|c|c|c|c|}
\hline & Sujets Carnets & Cercles de lecture & Entretien & Catégories \\
\hline Sujet 1 & $\begin{array}{l}\text { - Identification et } \\
\text { explicitation des } \\
\text { savoirs et des pratiques } \\
\text { enseignés }\end{array}$ & $\begin{array}{l}\text { - Formulation } \\
\text { des hypothèses } \\
\text { interprétatives }\end{array}$ & $\begin{array}{l}\text { - Identification } \\
\text { et explicitation } \\
\text { des savoirs et } \\
\text { des pratiques } \\
\text { enseignés }\end{array}$ & $\begin{array}{l}\text { - Maitrise des savoirs et des pratiques } \\
\text { enseignés } \\
\text { - } \quad \text { Lecture subjective sommaire } \\
\text { - } \quad \text { Développement de la réflexivité du } \\
\text { SLE (sujet lecteur enseignant) }\end{array}$ \\
\hline Sujet 2 & $\begin{array}{l}\text { - } \text { Reprise compréhensive } \\
\text { des savoirs enseignés } \\
\text { - Développement de la } \\
\text { subjectivité du lecteur } \\
\text { - Formulation } \\
\text { d'hypothèses } \\
\text { interprétatives }\end{array}$ & $\begin{array}{l}\text { - Prise en compte } \\
\text { de l'apport des } \\
\text { pairs dans le } \\
\text { développement de } \\
\text { la réflexivité }\end{array}$ & $\begin{array}{l}\text { - Analyse de son } \\
\text { parcours de } \\
\text { formation } \\
\text { - Verbalisation }\end{array}$ & $\begin{array}{l}\text { - } \text { Mobilisation des ressources } \\
\text { - } \quad \text { Qubjectives } \\
\text { - } \quad \text { Figure de l'auteur impliqué7 } \\
\text { - } \quad \text { Mise à distance des savoirs et des } \\
\text { pratiques enseignés en formation } \\
\text { Retour sur soi comme SLE }\end{array}$ \\
\hline Sujet 3 & $\begin{array}{l}\text { - } \text { Reprise compréhensive } \\
\text { des savoirs enseignés } \\
\text { - Transcription des } \\
\text { réponses du guide } \\
\text { d'entretien } \\
\text { - Formulation des } \\
\text { interprétations propres } \\
\text { sur le recueil }\end{array}$ & $\begin{array}{l}\text { - Activité } \\
\text { propice pour } \\
\text { l'interprétation des } \\
\text { textes }\end{array}$ & - Réflexivité du SLE & $\begin{array}{l}\text { - Mobilisation des ressources } \\
\text { subjectives et mise à distance de ses } \\
\text { interprétations } \\
\text { - } \quad \text { Mise à distance des savoirs } \\
\text { à enseigner et des pratiques } \\
\text { expérimentées en formation } \\
\text { - Retour sur soi comme lecteur et } \\
\text { comme enseignant }\end{array}$ \\
\hline Sujet 4 & $\begin{array}{l}\text { - Analyse du texte } \\
\text { en référence } \\
\text { aux ressources } \\
\text { épistémiques }\end{array}$ & $\begin{array}{l}\text { Compréhension } \\
\text { de soi comme } \\
\text { sujet lecteur et } \\
\text { interprétation des } \\
\text { textes }\end{array}$ & $\begin{array}{l}\text { - Faible activité } \\
\text { réflexive comme } \\
\text { lecteur } \\
\text { - } \quad \text { Impact négatif } \\
\text { des analyses } \\
\text { traditionnelles }\end{array}$ & $\begin{array}{l}\text { - } \text { Mobilisation des ressources } \\
\text { - } \text { subjectives } \\
\text { - } \text { - Qugure de l'auteur impliqué } \\
\text { - } \quad \text { Réflexivité du lecteur }\end{array}$ \\
\hline Sujet 5 & $\begin{array}{l}\text { - Développement de } \\
\text { la réflexivité comme } \\
\text { lecteur } \\
\text { - Reprise compréhensive } \\
\text { des savoirs enseignés }\end{array}$ & 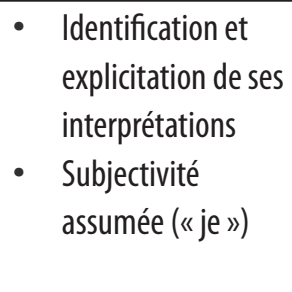 & $\begin{array}{l}\text { - Investissement } \\
\text { du lecteur } \\
\text { Projection } \\
\text { comme } \\
\text { enseignant }\end{array}$ & $\begin{array}{l}\text { - Mobilisation des ressources } \\
\text { subjectives } \\
\text { - Compréhension de soi comme sujet } \\
\text { lecteur } \\
\text { - Mise à distance des savoirs et des } \\
\text { pratiques enseignés en formation }\end{array}$ \\
\hline
\end{tabular}




\begin{tabular}{|c|c|c|c|c|}
\hline Sujet 6 & $\begin{array}{l}\text { - Identification, } \\
\text { explicitation et } \\
\text { évaluation des } \\
\text { interprétations }\end{array}$ & $\begin{array}{l}\text { - Forte activité } \\
\text { réflexive comme } \\
\text { sujet lecteur } \\
\text { - } \text { Posture de meneur } \\
\text { et gestion des } \\
\text { interactions }\end{array}$ & 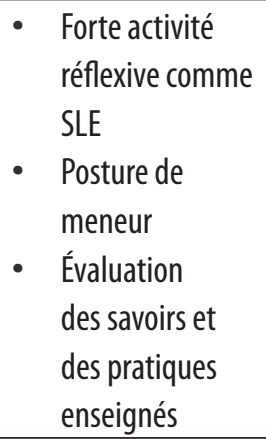 & $\begin{array}{l}\text { - Mise à distance de ses } \\
\text { interprétations } \\
\text { - } \quad \text { Figure de l'auteur impliqué } \\
\text { - Mise à distance des savoirs et } \\
\text { des pratiques expérimentées en } \\
\text { formation }\end{array}$ \\
\hline Sujet 7 & $\begin{array}{l}\text { - Forte activité réflexive } \\
\text { comme sujet lecteur } \\
\text { - Formulation } \\
\text { d'hypothèses } \\
\text { interprétatives } \\
\text { - Analyse de son parcours } \\
\text { interprétatif }\end{array}$ & $\begin{array}{l}\text { - Analyse des } \\
\text { poèmes en } \\
\text { référence aux } \\
\text { ressources } \\
\text { épistémiques }\end{array}$ & $\begin{array}{l}\text { Prise en compte } \\
\text { de l'apport des } \\
\text { pairs dans le } \\
\text { développement } \\
\text { de la réflexivité } \\
\text { du lecteur }\end{array}$ & $\begin{array}{l}\text { - Mise à distance de l'enseignement } \\
\text { - Questionnement du lecteur } \\
\text { - Compréhension de soi comme sujet } \\
\text { lecteur } \\
\text { - Mise à distance des savoirs et } \\
\text { des pratiques expérimentés en } \\
\text { formation }\end{array}$ \\
\hline Sujet 8 & $\begin{array}{l}\text { - Connaissances } \\
\text { linguistiques, } \\
\text { encyclopédiques et } \\
\text { rhétoriques } \\
\text { - Illustration de chaque } \\
\text { poème du recueil }\end{array}$ & $\begin{array}{l}\text { - Activité réflexive } \\
\text { allant de } \\
\text { l'identification } \\
\text { des hypothèses à } \\
\text { la validation des } \\
\text { interprétations } \\
\text { - Posture de meneur }\end{array}$ & & $\begin{array}{l}\text { - Mobilisation de ressources } \\
\text { - } \text { subjectives } \\
\text { - } \text { Puestionnement du lecteur } \\
\text { pairs de conscience du rôle des } \\
\text { réflexivité comme lecteur } \\
\text { - } \quad \text { Compréhension de soi comme sujet } \\
\text { lecteur } \\
\text { - Interprétation singulière du recueil }\end{array}$ \\
\hline
\end{tabular}

\section{Conclusions partielles}

Malgré sa timidité déclarée, le Sujet 1 s'est investi dans les différentes activités réalisées. Elle est d'avis qu'il faut maitriser les savoirs didactiques pour être un bon lecteur et un bon enseignant. C'est ce que nous observons dans ses discours, à travers la mise à distance des savoirs et des pratiques, qui témoigne ici du développement de la réflexivité comme SLE. Par ailleurs, nous pouvons avancer que les pairs ont fortement contribué au développement de la réflexivité de Libreville comme lecteur et à sa compréhension comme sujet lecteur.

S'agissant du Sujet 2, sa réflexivité comme SLE est réelle, car il met à distance les savoirs et les pratiques et analyse les transformations de son parcours de formation dans la durée (Abeme Ndong, 2018, p. 138). Toutefois, c'est la réflexivité du lecteur qui prédomine dans ses données, car il met à distance ses interprétations et opère un retour sur soi comme lecteur. Enfin, il semble que les activités collaboratives (cercle de lecture et entretien semi-dirigé) lui aient permis de s'ouvrir un peu plus et de produire des interprétations cohérentes. Ces activités apparaissent certainement moins formalistes que l'exploitation du carnet du lecteur qui pour ce sujet devrait «favoriser la verbalisation par laquelle le lecteur s'approprie le texte »(Ahr, 2010, p. 2-3). 
Pour le Sujet 3, il déclare se représenter comme un sujet lecteur grâce au dispositif et il confie qu'il faut amener le lecteur « au-delà des premières impressions » (Abeme Ndong, 2018, p. 147), ce qui l'amène à mobiliser diverses ressources. Par ailleurs, la prise en compte des savoirs et des pratiques enseignés et du rôle des pairs dans l'appropriation réflexive des savoirs et des pratiques le conduit au développement de la réflexivité comme SLE.

Le Sujet 4 confie que c'est durant l'entretien qu'il a trouvé satisfaction dans la formation à l'enseignement du français. Son évolution comme sujet lecteur l'amènera à produire des interprétations singulières. $\mathrm{Du}$ fait de ses croyances religieuses, il confie avoir été choqué par le premier poème du recueil à l'étude. Mais, au fil des pages, il s'est senti apaisé, ce qui a révélé sa sensibilité comme lecteur. D'ailleurs, ses interprétations des différents poèmes du recueil traduisent une forte mobilisation de ressources subjectives diverses (épistémiques, psychoaffectives, axiologiques et cognitives). En somme, la réflexivité du Sujet 4 comme lecteur et comme SLE trouve des échos significatifs dans le dispositif réalisé, car il confie qu'en plus de maitriser son objet, «l'enseignant doit être un sujet lecteur » (Abeme Ndong, 2018, p. 158).

Pour le Sujet 5, la réflexivité du lecteur est la catégorie dominante. Non seulement il mobilise diverses ressources durant ses interprétations, mais en plus la mise à distance de ses interprétations l'amène à la construction de soi comme lecteur. Par contre, sa réflexivité comme SLE reste sommaire, car, même s'il formule des reprises compréhensives des savoirs enseignés, il semble que leur appropriation ne soit pas effective lorsqu'il sous-entend encore que le lecteur est aussi libre durant la lecture méthodique que durant la lecture littéraire (Abeme Ndong, 2018, p. 161).

Au terme du dispositif, le Sujet 6 ne semble pas percevoir la différence entre la lecture méthodique et la lecture littéraire. À cet égard, ses interprétations du recueil témoignent d'une forte mobilisation de ressources épistémiques, car il cherche d'abord à appliquer ses connaissances sur l'analyse textuelle. Il apparait comme un cas « résistant ». Ses connaissances disciplinaires ne soutiennent pas la subjectivation contrairement à d'autres. Et, malgré son implication personnelle dans le cercle de lecture, sa réflexivité comme lecteur demeure au degré 1 (identification d'une hypothèse interprétative et explicitation), car selon luile sens ne se trouve que dans le texte.

Quant au Sujet 7, la réflexivité comme lecteur est observable de façon significative. En tant que sujet lecteur, elle confie s'appuyer particulièrement sur sa subjectivité, sur les ressources épistémiques et sur les pairs. Au niveau des ressources mobilisées, ce sont les ressources épistémiques qui dominent suivies des ressources psychoaffectives et des ressources cognitives. Dans ses contenus, elle ne se limite pas à la mise à distance de ses interprétations. Non seulement elle formule des interprétations personnelles, mais en plus, elle intègre les discours des pairs dans la production de ses interprétations. Le Sujet 7 a atteint un haut degré de réflexivité comme lecteur et comme SLE par la conceptualisation du processus interprétatif.

Enfin, le Sujet 8 se fait une représentation très claire des savoirs et des pratiques enseignés en formation. Selon lui, il faut accorder une place importante à celui qui lit le texte et qui va lui conférer un sens. Il confie que le dispositif lui a permis de réaliser qu'il est un sujet lecteur, car le sens se construit par rapport à la subjectivité du lecteur. Il prend conscience de sa subjectivité de lecteur, ce qui le conduit à effectuer des va-et-vient vers le texte pour produire une interprétation singulière. 
D’une part, ses données traduisent le développement de la réflexivité comme lecteur (mobilisation de ressources, questionnement du lecteur, dimension intersubjective de l'apprentissage). D'autre part, la mise à distance des savoirs et des pratiques, et la construction de soi comme FEF révèlent sa réflexivité comme SLE.

\section{Résultats et observations}

\section{Synthèse de l'analyse des données}

À la suite à notre collecte des données, nous avons accumulé une grande quantité de données avec les notes prises dans notre grille d'observation durant les séances dans les cercles, à travers les carnets des lecteurs et avec l'entretien semi-dirigé collectif mené au terme de la séquence didactique. Au terme de notre dispositif, les CL nous ont permis d'une part de mettre en avant l'autonomie des lecteurs et d'observer l'impact déterminant des interactions dans la production d'un texte singulier. D'autre part, à travers les CL, nous avons pu observer comment les FEF s'y prennent pour construire leur interprétation du texte. L'analyse de l'entretien semi-dirigé collectif révèle que les pratiques et les activités expérimentées dans ce dispositif ont reçu un avis favorable chez les FEF en formation à l'ENS au Gabon. Selon les participants, cette formation s'est avérée enrichissante, car elle leur a permis de développer des stratégies nouvelles pour mieux enseigner les textes littéraires aux élèves. Ainsi pour un des FEF, à travers ce dispositif d'enseignement, "l'élève s'exprime librement contrairement à une lecture méthodique ou analytique " (Abeme Ndong, 2018, p. 192). Il va plus loin en précisant que "l'élève apprend à relativiser son point de vue grâce au point de vue des autres lecteurs, une forme d'interactivité » (Abeme Ndong, 2018, p. 206). En somme, pour lui, il ne faut pas occulter la subjectivité du lecteur, car cela fait partie de la lecture littéraire.

Pour un autre, "l'investissement subjectif des lecteurs permet de donner libre cours à la relation du lecteur au texte et permet d'avoir une autre approche du texte et surtout l'épanouissement intellectuel du lecteur " (Abeme Ndong, 2018, p. 242). Cet étudiant conclut son propos en soulignant qu'en tant que futur enseignant, « il est dangereux de refuser le développement. Par conséquent, il faut se former à la lecture littéraire » (p. 206). Nous comprenons que les FEF considèrent que le dispositif expérimenté a contribué à les former à l'enseignement de la lecture littéraire. De même, les savoirs enseignés et à enseigner ont été assimilés, à tel point que la majorité des participants réalisent la différence entre la lecture méthodique et la lecture littéraire. Pour l'ensemble, il faut enseigner la lecture littéraire, car c'est le lecteur qui construit le sens du texte. Un des FEF déclare :

pour ma part, la lecture littéraire accorde la primauté au lecteur, elle est donc totalement différente de la lecture méthodique. Il y a une grande liberté chez le sujet lecteur du fait de son rapport particulier avec le texte. Alors que durant la lecture méthodique, quel que soit le rapport quion a avec le texte, on doit le ranger dans une certaine méthode. (p. 242) 


\section{Observations}

Nous avons observé que ce dispositif est en contradiction avec la formation scolaire et académique des FEF, qui est orientée vers l'analyse des indices textuels. Il ressort des données recueillies que les FEF ont du mal à faire abstraction de la figure du poète, plus exactement il semblerait que la subjectivité du lecteur s'exprime selon des modalités énonciatives traditionnelles «le poète dit que ». Ainsi, les interprétations sont formulées sous la forme des intentions attribuées au poète. Par exemple, en analysant le recueil, un FEF déclare :

à travers toutes ces expériences malheureuses de la vie, le poète se rend finalement compte qu'il ne doit pas s'en prendre à Dieu et comprend plutôt que c'est lui qui était dans une forme d'illusion (ou d'ignorance) de la réalité de l'existence; d'où son retour vers le père. (p. 172)

En substance, l'activité interprétative du sujet lecteur ne sénonce pas à la première personne, alors que dans les entretiens les FEF se considèrent majoritairement comme des sujets lecteurs. Cette contradiction méritera une analyse plus approfondie des données collectées, qui est encore en cours, au moment où nous écrivons ces lignes. La question du genre textuel serait également à explorer, puisque, pour certains participants, le genre lyrique a constitué un frein à l'expression de leur subjectivité de lecteur, car selon eux, le lyrisme est l'expression des sentiments personnels du poète.

\section{Les limites du dispositif}

\section{Réorganisation des séances}

Initialement, la séquence qui comportait huit séances de cours en incluant la séance préliminaire a été menée par l'enseignant formateur en sept séances. En effet, durant la séance préliminaire, l'enseignant formateur a constaté que les participants prenaient des notes sur des supports personnels, alors qu'il y avait des outils prévus pour cela. Aussi, le carnet de lecture leur a été remis durant la séance préliminaire. Cela a conduit l'enseignant formateur à combiner les séances 1 (le carnet de lecture) et 2 (constitution des CL). D'après notre grille d'observation, la séance 1 s'est déroulée en une heure de la manière suivante :

- lecture du fascicule de textes remis aux participants par des volontaires;

- mises au point théoriques par l'enseignant formateur;

- présentation du carnet lecture par l'enseignant formateur;

- échanges entre les participants et le formateur sur l'utilité du carnet de lecture;

- constitution des CL;

- règles d'interaction et de fonctionnement des CL.

Étant donné l'intérêt et la diversité des activités réalisées durant cette séance, l'entrée dans l'œuvre a constitué la séance 2. Nous avons observé pendant cette séance qui visait l'investissement subjectif des FEF que les responsables de cercles se chargeaient de distribuer la parole à chaque membre. Toutefois dans certains cercles, la posture de responsable de cercle a été assimilée à celle d'un enseignant maitre des lieux. Ainsi, un des FEF déclare : 
Je crois que comme sont les États, il faut une certaine organisation. Pour quion se comprenne dans ces activités de lecture, il faut quelqu'un qui puisse de temps en temps cadrer les discussions ou ramener l'ordre pour quion atteigne les objectifs de lecture. (ENT, p. 12)

Si l'on en vient à considérer les responsables de cercles comme des « chefs d'État ", nous craignons que cela n'empêche véritablement l'émergence des sujets lecteurs. D'ailleurs pour Giasson, l'enseignant doit demeurer un facilitateur, c'est-à-dire, « il a une tâche d'organisation et de soutien » (2014, p. 178). Il ne s'agit pas de reproduire les schémas de l'enseignement magistral.

Ensuite, notre dispositif proposait à la séance 4 (questionnement des textes) de travailler sur cinq poèmes choisis par l'enseignant formateur. Compte tenu de la difficulté pour les FEF de travailler sur cinq textes dans les CL en moins de deux heures, l'enseignant formateur a choisi trois poèmes à exploiter dans les CL. Les FEF ont trouvé que le temps alloué à l'interprétation des textes était trop court et qu'il valait mieux travailler sur un seul texte pour réaliser un travail collectif permettant à chacun de produire une interprétation singulière. Enfin, bien que les séances 6 (interprétation du recueil) et 7 (reconfiguration du texte) aient été menées par le formateur, nous pensons qu'elles auraient pu être combinées en une seule. Étant donné que l'interprétation du recueil avait été initiée à la séance 6, la reconfiguration du texte s'est organisée autour du débat sur les activités menées pendant la séquence didactique. Nous pensons que la séance 6 aurait pu viser l'interprétation collective du recueil par FEF dans les CL, tandis que la séance 7 aurait été orientée vers une interprétation singulière du recueil. La séquence didactique ainsi réorganisée se serait achevée par une évaluation des interprétations des FEF entre pairs.

\section{Contraintes méthodologiques}

Force est de constater aujourd'hui que la mise en œuvre des savoirs issus de la recherche rencontre des obstacles. Comment diffuser les dispositifs actuels et renouveler les démarches d'enseignement et d'apprentissage de la lecture littéraire? Ces dix dernières années, plusieurs chercheurs ont relevé les difficultés rencontrées dans l'enseignement de l'analyse des textes littéraires (Massol et ShawkyMilcent, 2011; Nguimbi, 2015; Rouxel, 2013). Ainsi, la récurrence des exercices traditionnels fondés sur l'acquisition et la transmission des savoirs est à l'origine de la difficulté des élèves et même des enseignants à se représenter comme sujets lecteurs. En regard de nos résultats, nous comprenons que faire place à la lecture subjective pour former des sujets lecteurs constitue un défi.

Par ailleurs, en choisissant l'entretien semi-dirigé collectif comme outil de collecte des données, nous voulions, entre autres, identifier les ressources mobilisées par les FEF. Finalement, le fait de mener un entretien collectif ne nous a pas laissé le temps suffisant pour revenir sur les questions posées et les réponses formulées par les participants.

\section{Conclusion}

Nous envisageons la lecture littéraire comme « un prodigieux moyen de stimuler la créativité des élèves, d'impulser des démarches interprétatives » (Rouxel, 2002, p. 22) conduisant à la formation d'un sujet lecteur actif et autonome, à même de comprendre et d'interpréter les textes littéraires. Pour la majorité des FEF, il faut enseigner la lecture littéraire aujourd'hui, car cette approche "permet à l'enseignant de 
s'effacer [...] pour mener l'élève à ne plus être passif et être constructeur de sa propre formation » (ENT, p. 29). Ce dispositif a révélé l'impact déterminant des interactions dans le processus de production d'un texte singulier. Au terme de cette recherche, il apparait que plusieurs FEF prennent conscience des transformations de leurs parcours de formation dans la durée. Cette prise de conscience de soi comme sujet lecteur se traduit par une interprétation singulière des textes. L'expérimentation d'une séquence didactique axée sur l'enseignement de la lecture littéraire semble contribuer à la formation de sujets lecteurs enseignants, mais cette approche théorique et didactique entre en tension avec les pratiques et les représentations issues des approches traditionnelles de la lecture scolaire.

\section{Notes}

1 Sauvaire montre que certaines activités telles la lecture, la relecture, la reconfiguration du texte initial amènent le lecteur vers une mise à distance des activités et des pratiques expérimentées en formation. Notons que la mise à distance est une des dimensions de la réflexivité.

2 Nous ne pouvions pas observer le déroulement des séances et enseigner simultanément. C'est la raison pour laquelle nous avons sollicité la participation d'un enseignant formateur pour la mise en œuvre de la séquence didactique.

3 Le versant herméneutique est axé sur la recherche du sens, de théories, de modèles. La démarche adoptée est interprétative, car le chercheur essaie de comprendre la réalité dans sa globalité.

4 Le versant praxéologique s'intéresse quant à lui à la transformation des pratiques et aux représentations des sujets étudiés. La démarche adoptée est soit exploratoire (exploration des possibles), soit actionnelle, soit évaluative (analyse des effets d'un dispositif).

5 Initialement, la séquence didactique comportait huit séances. Finalement lors de la réalisation de la séquence par l'enseignant formateur, la séance 7 a été supprimée, car elle semblait reprendre les activités de la séance 8.

6 Le poème « L'Ici », p. 47, a été choisi à l'unanimité.

7 Nous faisons de la notion de « auteur impliqué », une catégorie de la lecture et pas uniquement une catégorie de la rhétorique de la persuasion, car, c'est la manière dont le lecteur perçoit l'auteur. Le lecteur réel attribue spontanément le sens qu'il dégage du texte à l'auteur.

\section{Références}

Abeme Ndong, M. (2018). Formation des sujets lecteurs enseignants dans le secondaire au Gabon (Thèse de doctorat, Université Laval). Repéré à https://corpus.ulaval.ca/jspui/bitstream/20.500.11794/31149/1/34391.pdf

Ahr, S. (2010). « D'une lecture empirique à une lecture subjective argumentée : quels processus cognitifs et langagiers mobiliser? ", 11e Rencontres des chercheurs en didactique des littératures, Genève, mars 2010.

Ahr, S. (2013). De quelques usages du carnet/journal de lecteur/lecture dans le second degré.

Dans S. Ahr et P. Joole (dir.), Carnet/journal de lecteur/lecture. Quels usages, pour quels enjeux, de l'école à l'université? (p. 4153). Namur : Presses universitaires de Namur.

Ahr, S. (2014). L'enseignement de la littérature aux cycles 3 et $4:$ quelles orientations pour quels enjeux? Repéré à https://www. education.gouv.fr/sites/default/files/imported files/document/AHR Sylviane - MCF - CSP Contribution 362982. pdf

Assoumou-Mombe, M.-C. (2011). Rapport sur l'enseignement du français au Gabon. Libreville : Département de français.

Bayard, P. (1998). Qui a tué Roger Ackroyd? Paris : Les Éditions de Minuit.

Bucheton, D., Alexandre, D. et Jurado, M. (2014). Refonder l'enseignement de l'écriture vers des gestes professionnels plus ajustés du primaire au lycée. Paris, France : Retz. 
Burdet, C. et Guillemin, S. (2013). Les cercles de lecture : un dispositif favorisant la gestion de la compréhension et l'autonomie du lecteur. Forumlecture.ch, 2013(1), 1-22. Repéré à https:/www.forumlecture.ch/sysModules/obxLeseforum/Artike1/483/2013 1 Burdet-Guillemin.pdf

Chabanne, J.-C. et Bucheton, D. (2002). La réflexivité : une notion à définir et à discuter. Dans J.-C. Chabanne et D. Bucheton (dir.), Parler et écrire pour penser, apprendre et se construire. L'écrit et l'oral réflexifs (p. 3-23). Paris : Presses universitaires de France.

Deronne, C. (2011). Former des enseignants à exprimer leur expérience singulière d'une œuvre littéraire ou plastique. Repères, (43), 103-126. http://dx.doi.org/10.4000/reperes.222

Dufays, J.-L. (2010). Au carrefour de trois méthodologies : une recherche en didactique de la lecture littéraire. Dans L. Paquay, M. Crahay et J.-M. De Ketele, L'analyse qualitative en éducation. Des pratiques de recherche aux critères de qualité. Hommage à Michael Huberman (2 éd., p. 143-164). Louvain-la-Neuve : De Boeck.

Dufays, J.-L. (2013). Sujet lecteur et lecture littéraire : quelles modélisations pour quels enjeux? Recherches et Travaux, (83), 77-88. Repéré à http://Recherchestravaux.revues.org/666

Dufays, J.-L., Gemenne, L. et Ledur, D. (2005). Pour une lecture littéraire : histoire, théories, pistes pour la classe (2éd.). Bruxelles : De Boeck.

Émery-Bruneau, J. (2010). Le rapport à la lecture littéraire : des pratiques et des conceptions de sujets-lecteurs en formation à l'enseignement du français à des intentions didactiques (Thèse de doctorat, Université Laval). Repéré à https://corpus.ulaval.ca/jspui/bitstream/20.500.11794/21444/1/27627.pdf

Émery-Bruneau, J. (2011). Former des «sujets-lecteurs-enseignants » : une responsabilité collective pour des actions didactiques réfléchies. Québec français, (163), 79-81. Repéré à https://www.erudit.org/fr/revues/qf/2011-n163-qf1823256/65428ac/

Engouang, H. (2011). Les temps déchirés. Libreville : Les Éditions Ntsame.

Fourtanier, M.-J., Langlade, G. et Mazauric, C. (2006, avril). Dispositifs de lecture et formation des lecteurs. Communication présentée aux 7èmes Rencontres des chercheurs en didactique de la littérature, IUFM de Montpellier. Repéré à https://halshs.archives-ouvertes.fr/halshs-00522189

Giasson, J. (2014). Les textes littéraires à l'école. Louvain-la-Neuve : De Boeck.

Langlade, G. (2004a). Sortir du formalisme, accueillir les lecteurs réels. Le français aujourd'bui, (145), 85-96. http://dx.doi.org/10.3917/1fa.145.0085

Langlade, G. (2004b). Le sujet lecteur auteur de la singularité du texte. Dans A. Rouxel et G. Langlade (dir.), Le sujet lecteur : lecture subjective et enseignement de la littérature (p. 81-91). Rennes : Presses universitaires de Rennes.

Langlade, G. et Fourtanier, M.-J. (2007). La question du sujet lecteur en didactique de la lecture littéraire. Dans E. Falardeau, C. Fisher, C. Simard et N. Sorin (dir.), La didactique du français : les voies actuelles de la recherche (p. 101123). Québec, QC : Presses de l'Université Laval.

Louichon, B. (2011). La lecture littéraire est-elle un concept didactique? Dans B. Daunay, Y. Reuter et B. Schneuwly (dir.), Les concepts et les méthodes en didactique du français (p. 195-216). Namur : Presses universitaires de Namur.

Makaya, A. (2013). La formation initiale des enseignants au Gabon : quels effets sur les conceptions de futurs enseignants de trois cohortes successives? (Thèse de doctorat, Université catholique de Louvain). Repéré à https://dial.uclouvain.be/pr/boreal/object/boreal:132059

Massol, J.-F. et Shawky-Milcent, B. (2011). Texte du lecteur et commentaire de texte : relations, évolutions, modalités d'apprentissage. Dans C. Mazauric, M.-J. Fourtanier, et G. Langlade (dir.), Textes de lecteurs en formation (vol. 3, p. 231244). Bruxelles : Peter Lang.

MEN. (2018, 7 mars). Arrêté no 00058/MEN/SG/DGESN portant institution de l'approche par les compétences de base et des nouveaux processus d'évaluation des acquis scolaires de l'enseignement secondaire général et technique. Repéré à https://64695626-4c16-4c32-a051-0af78a00c561.filesusr.com/ugd/33bbf3 23d67183e3004cfc91128291df736dfd.pdf 
MEN et IPN. (1985/2012). L’enseignement du français au second cycle. Libreville : Département de français.

Nguimbi, A. (2015). Discours et pratiques sur l'enseignement des textes littéraires au lycée. Libreville, Gabon : Odem.

Ouellet, S. (2012). Le sujet lecteur et scripteur : développement d'un dispositif didactique en classe de littérature (Thèse de doctorat, Université de Toulouse II - Le Mirail). Repéré à https://archipel.uqam.ca/4675/1/D2324.pdf

Ricœur, P. (1985). Temps et récit. 3. Le temps raconté. Paris : Éditions du Seuil.

Rouxel, A. (1996). Enseigner la lecture littéraire. Rennes : Presses universitaires de Rennes.

Rouxel, A. (2002). Qu'entend-on par lecture littéraire? Dans C. Tauveron (dir.), La lecture et la culture littéraires au cycle des approfondissements (p. 12-22). Repéré à https://media.eduscol.education.fr/file/Formation continue enseignants/14/7/ lecture culture litteraires 111147.pdf

Rouxel, A. (2013). L'avènement du sujet lecteur. Avancées scientifiques et perspectives pour l'enseignement de la littérature. Dans S. Ahr et P. Joole (dir.), Carnet/journal de lecteur/lecture : quels usages, pour quels enjeux, de l'école a囚 l'université? (p. 115-128). Namur : Presses universitaires de Namur.

Rouxel, A. et Langlade, G. (2004). Le sujet lecteur : lecture subjective et enseignement de la littérature. Rennes : Presses universitaires de Rennes.

Sauvaire, M. (2013). Diversité des lectures littéraires. Comment former des sujets lecteurs divers? (Thèse de doctorat, Université Laval). Repéré à https://corpus.ulaval.ca/jspui/bitstream/20.500.11794/24045/1/29976.pdf

Sauvaire, M. (2015). La diversité des sujets lecteurs dans l'enseignement de la lecture littéraire. Éducation E̊ Didactique, 9(2), 107-117. http://dx.doi.org/10.4000/educationdidactique.2310

Tauveron, C. (1999). Comprendre et interpréter le littéraire à l'école : du texte réticent au texte proliférant. Repères, (19), 9-38. http://dx.doi.org/10.3406/reper.1999.2289

Tauveron, C. (2007). Le texte singulier de l'élève ou la question du sujet scripteur. Le français aujourd'hui, (157), 75-82. http://dx.doi.org/10.3917/1fa.157.0075

Terwagne, S., Vanhulle, S. et Lafontaine, A. (2003). Les cercles de lecture : interagir pour développer ensemble des compétences de lecteurs. Bruxelles : De Boeck.

Vanhulle, S. (2009). Des savoirs en jeu aux savoirs en «je». Cheminements réflexifs et subjectivation des savoirs chez de jeunes enseignants en formation. Berne : Peter Lang.

Vibert, A. (2013). Faire place au sujet lecteur en classe : quelles voies pour renouveler les approches de la lecture analytique au collège et au lycée?. Repéré à

https://eduscol.education.fr/lettres/im pdflettres/intervention-anne-vibert-lecture-vf-20-11-13.pdf

\section{Pour citer cet article}

Abeme Ndong, M. (2020). Dispositif de formation de sujets lecteurs enseignants du secondaire. Formation et profession, 28(1), 110-126. http://dx.doi.org/10.18162/fp.2020.501 